\title{
A multilocus sequence analysis approach to the phylogeny and taxonomy of the Halobacteriales
}

Correspondence
R. Thane Papke
thane@uconn.edu

\author{
R. Thane Papke, ${ }^{1}$ Emma White, ${ }^{1}$ Prajwal Reddy, ${ }^{1}$ Griffin Weigel, ${ }^{1}$ \\ Masahiro Kamekura, ${ }^{2}$ Hiroaki Minegishi, ${ }^{3}$ Ron Usami ${ }^{3}$ \\ and Antonio Ventosa ${ }^{4}$
}

\author{
${ }^{1}$ Department of Molecular and Cell Biology, University of Connecticut, Storrs, CT 06269, USA \\ ${ }^{2}$ Halophiles Research Institute, Noda, Chiba 278-0043, Japan \\ ${ }^{3}$ Bio-Nano Electronics Research Center, Toyo University, Kujirai, Kawagoe, Saitama 350-8585, \\ Japan \\ ${ }^{4}$ Departamento de Microbiologia y Parasitologia, Facultad de Farmacia, Universidad de Sevilla, \\ 41012 Sevilla, Spain
}

\begin{abstract}
Members of the order Halobacteriales are obligate extreme halophiles that belong to the domain Archaea. The classification of the Halobacteriales currently relies on a polyphasic approach, which integrates phenotypic, genotypic and chemotaxonomic characterization. However, the most utilized genetic marker for phylogeny, the 16S rRNA gene, has multiple drawbacks for use with the Halobacteriales: the species of many genera exhibit large intragenic differences between multiple ribosomal RNA operons, the gene is too conserved to discriminate reliably at the species level and it appears to be the most frequently recombined gene between closely related species. Moreover, the Halobacteriales is a rapidly expanding group due to recent successes at cultivating novel strains from a diverse set of hypersaline environments; a fast, reliable, inexpensive, portable molecular method for discriminating species is required for their investigation. Recently, multilocus sequence analysis (MLSA) has been shown to be an effective tool for strain identification and taxonomic designation, even for those taxa that experience frequent lateral gene transfer and homologous recombination. In this study, MLSA was utilized for evolutionary and taxonomic investigation of the Halobacteriales. Efficacy of the MLSA approach was tested across a hierarchical gradient using 52 halobacterial strains, representing 33 species (including names without standing in nomenclature) and 14 genera. A subset of 21 strains from the genus Haloarcula was analysed separately to test the sensitivity and relevance of the MLSA approach among closely related strains and species. The results demonstrated that MLSA differentiated individual strains, reliably grouped strains into species and species into genera and identified potential novel species and also family-like relationships. This study demonstrates that MLSA is a rapid and informative molecular method that will probably accommodate strain analysis at any taxonomic level within the Halobacteriales.
\end{abstract}

\section{INTRODUCTION}

Members of the order Halobacteriales are aerobic, heterotrophic and obligately halophilic archaea. Commonly known as haloarchaea, these halophiles are found in environments with a minimum salt concentration of $1.5 \mathrm{M}$, such as salt lakes, soda flats, hypersaline soils, underground salt deposits

Abbreviations: $\mathrm{AU}$, approximately unbiased; LGT, lateral gene transfer; $M L$, maximum-likelihood; MLSA, multilocus sequence analysis; MP, maximum-parsimony.

The GenBank/EMBL/DDBJ accession numbers for the sequences reported in this study are detailed in Table 1.

A supplementary table is available with the online version of this paper. and evaporation ponds for the production of sea salt (Oren, 1994). Furthermore, they are the dominant members in those communities. In situ community characterization of hypersaline environments as typically revealed through $16 \mathrm{~S}$ rRNA gene sequences (Casamayor et al., 2002), protein markers (Papke et al., 2003; Pasić et al., 2007) and, more recently, metagenomics (Bodaker et al., 2010; CuadrosOrellana et al., 2007; Legault et al., 2006) has discovered a surprising amount of haloarchaeal diversity, which is reflected in the growing number of described species and genera (Ochsenreiter et al., 2002; Oren, 2008). As of July 2010, 29 genera and 101 species names are recognized (http:// www.the-icsp.org/taxa/halobacterlist.htm). 
Use of the 16S rRNA gene sequence to assess phylogenetic relationships is universal in taxonomic descriptions of the Halobacteriales. However, due to the highly conserved nature of the 16S rRNA gene [e.g. only five polymorphisms among 1473 positions between Haloferax volcanii and Haloferax alexandrinus (Asker \& Ohta, 2002)], sequence data cannot be used reliably to differentiate closely related species, and evidence suggests that it is the most frequently transferred gene among close relatives (Papke, 2009), which confounds species identification. Additionally, it was discovered that single strains from many genera of the Halobacteriales, including Haloarcula, Halosimplex and Natrinema, have highly divergent, paralogous rRNA operons that exhibit over $6 \%$ sequence divergence, several indels and extensive evidence for intragenic and interspecies recombination (Boucher et al., 2004; López-López et al., 2007), making phylogeny and taxonomy difficult when based on this marker alone. Because of these complications, the essential single-copy RNA polymerase $r p o B^{\prime}$ was proposed to complement or replace $16 \mathrm{~S}$ rRNA gene analysis (Case et al., 2007; Enache et al., 2007; Minegishi et al., 2010). However, single-gene phylogenies are limited to the evolution of the analysed gene, which, in the light of lateral gene transfer (LGT), probably does not correspond to the cumulative evolutionary history of the investigated organisms. For instance, comparison of $16 \mathrm{~S}$ rRNA and $r p o B^{\prime}$ gene phylogenies among taxa of the Halobacteriales revealed that the overall phylogenetic topologies were incongruent (Walsh et al., 2004).

A recently developed molecular method for taxonomy, called multilocus sequence analysis (MLSA), sidesteps many of the limitations of single-gene phylogenies by using four to 12 concatenated gene fragments and imparts an averaged phylogeny of those loci. By focusing on gene segments, rapid and rigorous analysis for taxonomic purposes is facilitated (Maiden et al., 1998). Though MLSA was originally designed for epidemiological studies, it was demonstrated to be robust for circumscribing species and assessing their evolutionary history. MLSA was effective for identifying species clusters of highly recombinogenic Neisseria populations (Hanage et al., 2005), while also demonstrating evolutionary trends of 'despeciation' in rapidly evolving populations of Campylobacter jejuni and C. coli (Sheppard et al., 2008). Furthermore, it can be used to differentiate between closely related strains and species that exhibit little or no 16S rRNA gene sequence divergence (Papke et al., 2004, 2007).

Applied to the genus Halorubrum of the Halobacteriales, an MLSA approach led to the discovery of three well-supported, highly recombinogenic, species-like phylogenetic clusters that were identical or nearly identical at the $16 \mathrm{~S}$ rRNA gene locus (Papke et al., 2007). In view of the potential strength of MLSA for identifying and classifying species of the Halobacteriales, it is important to test the protocol across a large diversity of species and genera. Therefore, the main purpose of this study was to develop an MLSA approach for the Halobacteriales that could be utilized for all current and future described species.

\section{METHODS}

Strains. Twenty-one closely related strains from seven species whose names have been validly published belonging to the genus Haloarcula and an additional group of 31 strains representing 13 other genera in the order Halobacteriales were analysed in this study (Table 1). Strains of species with validly published names were cultivated in media recommended by the online catalogue of the Japan Collection of Microorganisms (JCM; http://www.jcm.riken.jp/). Strains of species without validly published names or that have not been deposited in culture collections were grown in media listed in Supplementary Table S1, available in IJSEM Online.

Amplification and sequencing. Genomic DNA from cultures was isolated using standard methods (e.g. Qiagen kit), quantified and checked for purity using a Nanodrop spectrophotometer ND-1000 at $260 / 280 \mathrm{~nm}$ and diluted with $1 \mathrm{M}$ Tris/HCl to $20 \mathrm{ng} \mu \mathrm{l}^{-1}$ for subsequent PCR analysis. From each strain, five gene fragments were amplified and sequenced: $a t p B, E F-2, r a d A, r p o B^{\prime}$ and $\sec Y$. These genes were chosen for analysis because they are housekeeping genes that were previously investigated in halobacteria (e.g. Kamaishi et al., 1996; Minegishi et al., 2010; Nakamura et al., 1999; Renesto et al., 2001; Sandler et al., 1999). The PCR and sequencing primers used in this study were adapted from Papke et al. (2004, 2007) to accommodate a wider taxonomic range (Table 2). Names of primers include information on the region amplified for each gene.

Amplification was performed with Invitrogen Taq DNA Polymerase Native or Roche FastStart Universal SYBR Green Master (Rox). For the majority of loci, the following recipe was used: $5 \mu l 10 \times$ buffer, $1.5 \mu \mathrm{l} \mathrm{MgCl}_{2}$ (50 mM stock), $1 \mu \mathrm{l}$ dNTPs, $2.0 \mu \mathrm{l}$ each primer $(10 \mu \mathrm{M}), 1 \mu \mathrm{l}$ Taq polymerase $\left(5 \mathrm{U}^{-1}\right), 36.5 \mu \mathrm{l}$ distilled water and $1 \mu \mathrm{l}$ template $\left(20 \mathrm{ng}^{-1} \mathrm{l}^{-1}\right)$. The $\sec Y$ gene primers were added as $3.0 \mu \mathrm{l}$ aliquots $(10 \mu \mathrm{M}$ stock). All reactions were performed in an Eppendorf Mastercycler EPgradient thermocycler. The annealing temperature for the thermal profile was optimized for each primer set (Table 2). The general thermal profile included an initial denaturation step $\left(1 \mathrm{~min}, 94^{\circ} \mathrm{C}\right)$ followed by 30 cycles of denaturation $(1 \mathrm{~min}$, $\left.94{ }^{\circ} \mathrm{C}\right)$, annealing $(1 \mathrm{~min})$ and extension $\left(1 \mathrm{~min}, 72{ }^{\circ} \mathrm{C}\right)$ and a final extension period $\left(5 \mathrm{~min}, 72^{\circ} \mathrm{C}\right)$.

PCR amplicons were purified using standard procedures and sequenced in both directions using BigDye chemistry and an $\mathrm{ABi}$ $3130 x$ l DNA Analyzer or an ABi 3730xl DNA Analyzer through Genewiz (http://www.Genewiz.com). Sequencing primers are listed in Table 2. Each sequence was edited using the software package Geneious (http://www.geneious.com/). Amplicon lengths in nucleotide base pairs are: atpB, 493; $E F-2,507$; radA, 498; rpoB', 519; secY, 495.

Multisequence alignment. DNA sequences were translated into amino acid sequences, aligned using Muscle version 3.6 (Edgar, 2004a, b) and edited using Mesquite version 2.71 (Maddison \& Maddison, 2009). Nucleotide sequences from Haloarcula strains were aligned separately using the amino acid alignment as a template. Regions of sequence alignments present in less than $50 \%$ of the taxa were removed for phylogenetic analysis. Alignments of each gene for both the comprehensive Halobacteriales dataset and the Haloarcula subset were concatenated in the following order: atpB, $E F-2, \operatorname{rad} A$, $r p o B^{\prime}$ and secY. Alignments are available upon request from the corresponding author.

Phylogenetic reconstruction and congruence. Phylogenetic reconstructions for the Halobacteriales dataset and the Haloarcula subset were performed on each gene and the concatenated loci. Optimal models of evolution were estimated from the nucleotide data of the Haloarcula subset using jModelTest version 0.1.1 (Guindon \& Gascuel, 2003; Posada, 2008), considering 11 substitution types, and from the amino acid data of the Halobacteriales dataset using ProtTest 
Table 1. Analysed strains of the Halobacteriales and other archaea, with accession numbers

Genera are abbreviated following the recommendations of the ICSP Subcommittee on the taxonomy of Halobacteriaceae (Oren \& Ventosa, 2000) as follows: Har., Haloarcula; Hbt., Halobacterium; Hcc., Halococcus; Hfx., Haloferax; Hmc., Halomicrobium; Hqr., Haloquadratum; Hrd., Halorhabdus; Hrr., Halorubrum; Htg., Haloterrigena; Nab., Natrialba; Nnm., Natrinema; Nbt., Natronobacterium; Nmn., Natronomonas; Nrr., Natronorubrum. ND, Not determined (unable to obtain a PCR product). Accession numbers from this study begin HQ.

\begin{tabular}{|c|c|c|c|c|c|}
\hline Strain & $\operatorname{atpB}$ & $E F-2$ & $\operatorname{radA}$ & $r p o B^{\prime}$ & $\sec Y$ \\
\hline Har. amylolytica JCM $13557^{\mathrm{T}}$ & HQ149372 & HQ149388 & HQ149431 & $\mathrm{AB} 477142$ & HQ149499 \\
\hline 'Har. californiae' JCM 8912 & HQ149343 & HQ149390 & HQ149433 & $\mathrm{AB} 477144$ & HQ149501 \\
\hline Har. hispanica ATCC $33960^{\mathrm{T}}$ & HQ149344 & HQ149391 & HQ149434 & HQ149480 & HQ149502 \\
\hline Har. japonica JCM $7785^{\mathrm{T}}$ & HQ149345 & HQ149392 & HQ149435 & $\mathrm{AB} 477146$ & HQ149503 \\
\hline Har. quadrata JCM $11048^{\mathrm{T}}$ & HQ149348 & HQ149395 & HQ149438 & HQ149482 & HQ149504 \\
\hline 'Har. sinaiiensis' ATCC 33800 & HQ149350 & HQ149397 & HQ149440 & HQ149483 & HQ149505 \\
\hline Haloarcula sp. Aidin-2 & HQ149336 & HQ149382 & HQ149425 & HQ149471 & HQ149494 \\
\hline Haloarcula sp. Aidin-5 & HQ149337 & HQ149383 & HQ149426 & HQ149472 & HQ149495 \\
\hline Haloarcula sp. Aidin-9 & HQ149338 & HQ149384 & HQ149427 & $\mathrm{ND}$ & HQ149496 \\
\hline Haloarcula sp. Aidin-11 & HQ149335 & HQ149381 & HQ149424 & HQ149470 & HQ149493 \\
\hline Haloarcula sp. Toen-14 & HQ149368 & HQ149422 & HQ149467 & HQ149478 & HQ149522 \\
\hline Haloarcula sp. Toen-15 & HQ149369 & HQ149423 & HQ149468 & HQ149479 & HQ149523 \\
\hline Haloarcula sp. XD1 & HQ149340 & HQ149386 & HQ149429 & ND & HQ149497 \\
\hline Har. vallismortis ATCC $29715^{\mathrm{T}}$ & HQ149351 & HQ149398 & HQ149441 & AB477148 & HQ149506 \\
\hline Hbt. salinarum JCM 9120 & HQ149380 & $\mathrm{X} 17148$ & AF090196 & X57144 & HQ149507 \\
\hline Hbt. salinarum NCIMB 2288 & AM774415 & HQ149399 & HQ149442 & CAP15004 & HQ149508 \\
\hline Halobacterium sp. NRC-1 & AE004437 & AE004437 & AE004437 & AAG20693 & AE004437 \\
\hline Hcc. hamelinensis JCM $12892^{\mathrm{T}}$ & HQ149373 & HQ149400 & HQ149443 & AB477156 & HQ149509 \\
\hline Hcc. morrhuae JCM $8876^{\mathrm{T}}$ & HQ149352 & HQ149401 & HQ149444 & HQ149484 & HQ149510 \\
\hline Hcc. saccharolyticus ATCC $49257^{\mathrm{T}}$ & HQ149353 & HQ149402 & HQ149445 & HQ149485 & HQ149511 \\
\hline Hfx. denitrificans ATCC $35960^{\mathrm{T}}$ & HQ149354 & HQ149403 & HQ149446 & HQ149486 & HQ149512 \\
\hline Hrr. lacusprofundi ATCC $49239^{\mathrm{T}}$ & HQ149375 & NC_012029 & HQ149453 & AB477181 & NC_012029 \\
\hline Hrr. saccharovorum JCM $8865^{\mathrm{T}}$ & HQ149376 & HQ149409 & HQ149454 & AB477184 & HQ149516 \\
\hline Hrr. sodomense JCM $8880^{\mathrm{T}}$ & HQ149377 & HQ149410 & $\mathrm{ND}$ & AB477185 & HQ149517 \\
\hline Halorubrum sp. ТР009 & AM777270 & AM777546 & AM777664 & HQ149489 & HQ149513 \\
\hline Hrr. vacuolatum JCM $9060^{\mathrm{T}}$ & HQ149378 & HQ149411 & $\mathrm{ND}$ & AB477190 & HQ149518 \\
\hline Htg. turkmenica GSL-11 & HQ149359 & HQ149412 & HQ149455 & HQ149492 & ND \\
\hline Htg. turkmenica ATCC $51198^{\mathrm{T}}$ & HQ149360 & HQ149413 & HQ149456 & AB477198 & NC_013743 \\
\hline Natrialba sp. Aidin-3 & HQ149361 & HQ149414 & HQ149457 & HQ149476 & HQ149520 \\
\hline Nab. magadii ATCC $43099^{\mathrm{T}}$ & HQ149379 & NC_013922 & HQ149458 & AB477206 & NC_013922 \\
\hline Nnm. pallidum NCIMB 784 & HQ149363 & HQ149418 & HQ149462 & $\mathrm{AB} 477210$ & ND \\
\hline Natrinema sp. XA3-1 & HQ149362 & HQ149417 & HQ149461 & AB295622 & HQ149524 \\
\hline Nnm. versiforme JCM $10478^{\mathrm{T}}$ & HQ149364 & ND & HQ149463 & $\mathrm{AB} 477212$ & HQ149519 \\
\hline Nbt. gregoryi NCIMB $2189^{\mathrm{T}}$ & HQ149371 & HQ149416 & HQ149460 & AB477213 & $\mathrm{ND}$ \\
\hline Natronobacterium sp. SSL6 & HQ149370 & HQ149415 & HQ149459 & AJ809525 & $\mathrm{ND}$ \\
\hline Nmn. pharaonis JCM $8858^{\mathrm{T}}$ & NC_007426 & NC_007426 & NC_007426 & AJ809526 & NC_007426 \\
\hline Natronorubrum sp. JCM 10938 & HQ149365 & HQ149419 & HQ149464 & AJ809527 & $\mathrm{ND}$ \\
\hline
\end{tabular}


Table 1. cont.

\begin{tabular}{|c|c|c|c|c|c|}
\hline Strain & $\operatorname{atpB}$ & $E F-2$ & $\operatorname{radA}$ & $r p o B^{\prime}$ & $\sec Y$ \\
\hline Natronorubrum sp. JCM 10944 & HQ149366 & HQ149420 & HQ149465 & ND & ND \\
\hline Archaeoglobus fulgidus DSM $4304^{\mathrm{T}}$ & NC_000917 & NC_000917 & NC_000917 & NC_000917 & NC_000917 \\
\hline Methanosarcina barkeri Fusaro & СР000099 & СР000099 & СР000099 & СР000099 & СР000099 \\
\hline Methanosarcina mazei Go1 & AE008384 & AE008384 & AE008384 & AE008384 & AE008384 \\
\hline Methanothermobacter thermautotrophicus $\Delta \mathrm{H}^{\mathrm{T}}$ & СР002278 & СР002278 & СР002278 & СР002278 & СР002278 \\
\hline
\end{tabular}

version 2.4 (Abascal et al., 2005; Guindon \& Gascuel, 2003) with the slow optimization strategy. Phylogenies reconstructed with maximum-likelihood (ML) mapping used RAxML version 7.0.4 (Stamatakis, 2006) with 100 bootstrap replicates. These phylogenies were based on the GTR nucleotide model of evolution and the WAG amino acid model of evolution. The inclusion of an alpha parameter and invariable sites was determined by the results of jModelTest version 0.1.1 and ProtTest version 2.4, respectively.

Additional support for ML phylogenies was determined through bootstrap analysis with 100 pseudoreplicates using distance and maximum-parsimony (MP) criteria, which were calculated using PAUP $^{*}$ version $4.0 \mathrm{~b} 10$ (Swofford, 2003). The evolutionary models for both distance and MP criteria were recommended by the results from jModelTest version 0.1.1 and ProtTest version 2.4. All bootstrap values equal to or greater than $70 \%$ that corresponded to clades represented in the ML phylogenies were mapped onto those trees.

Optimal substitution models were approximated from the nucleotide data from the Haloarcula strains using jModelTest and from the amino acid data from all of the strains in this study using ProtTest; the results of these tests are shown in Table 3. The models proposed for the nucleotide data included TN (Tamura \& Nei, 1993), TIM2 and TIM3, and GTR (Tavaré, 1986). These models all consider unequal base frequencies and two different transition rates, but vary in the number of transversion rates deemed necessary to model evolution.

Table 2. Amplification and sequencing primers

Primer names are derived from the locus they amplify, followed by the position in the alignment that they prime, whether they were forward (F) or reverse (R) and whether they were used for sequencing (seq) or had polylinker (poly) or M13 sequences used for sequencing. $T_{\mathrm{A}}$ is the annealing temperature as determined by gradient PCR. M13 and polylinker sequences are indicated in bold.

\begin{tabular}{|c|c|c|}
\hline Primer & $T_{\mathrm{A}}\left({ }^{\circ} \mathrm{C}\right)$ & Sequence $\left(5^{\prime}-3^{\prime}\right)$ \\
\hline atpB_906Ra & 54 & GCCAGGTCVGTRTACATGTA \\
\hline atpB_906Rb_poly & 60 & GACGTCGCATGCTGAATTCATAGATGCCGGGCCAGGTCVGTRTACATGTA \\
\hline atpB_409Fb_seq & - & GACATCGTCGGTGAGSCVATSAACCC \\
\hline atpB_906Rb_seq & - & GCCAGGTCVGTRTACATGTA \\
\hline EF-2_1147Ra & 56 & GTGGTCGATGGWYTCGAAHGG \\
\hline EF-2_634Fb_poly & 58 & TGAATTCATAGATGCCGGGGGTCCGCGCTVTACAARTGG \\
\hline EF-2_1147Rb_poly & 58 & TGAATTCATAGATGCCGGGATGTGGTGATSGACTCGAASGG \\
\hline EF-2_634Fb_seq & - & TCCGCGCTVTACAARTGG \\
\hline EF-2_1147Rb_seq & - & GTGGTCGATSGACTCGAASGG \\
\hline radA_prSJS252Fa & 54 & ACSGARKTSTWCGGSGARTTCGGSKCSGGSAA \\
\hline rpoB'_1462Ra & 56 & AGCGGGCGTGSAKYTTGTT \\
\hline rpoB'_962Fb_M13 & 58 & TGTAAAACGACGGCCAGTTCGAAGAGCCGGACGACATGG \\
\hline rpoB'_1504Rb_M13 & 58 & CAGGAAACAGCTATGACCGGTCAGCACCTGBACCGGNCC \\
\hline secY_356Fa & 53 & TCTATCAGGGVCKNCAGAAG \\
\hline secY_856Ra & 53 & GCRTARATGAGCTTBACCGGG \\
\hline secY_356Fb_poly & 60 & GACGTCGCATGCTGAATTCATAGATGCCGGTCTATCAGGGVCKNCAGAAG \\
\hline secY_856Rb_poly & 60 & ACGTCGCATGCTGAATTCATAGATGCCGGGCRTARATGAGCTTBACCGGG \\
\hline secY_856Rc_M13 & 60 & CAGGAAACAGCTATGACGCTTACATGAGCTTBACCGGG \\
\hline secY_356Fb_seq & - & TCTATCAGGGVCKNCAGAAG \\
\hline
\end{tabular}


Table 3. jModelTest- and ProtTest-determined evolutionary models used for constructing the phylogeny of each locus and dataset

\begin{tabular}{|lcc|}
\hline Locus & $\begin{array}{c}\text { Haloarcula } \\
\text { (jModelTest) }\end{array}$ & $\begin{array}{c}\text { Halobacteriales } \\
\text { (ProtTest) }\end{array}$ \\
\hline Concatenated & $\mathrm{TN}+\Gamma+\mathrm{I}$ & $\mathrm{LG}+\Gamma+\mathrm{I}$ \\
atpB & $\mathrm{GTR}+\Gamma+\mathrm{I}$ & $\mathrm{LG}+\Gamma+\mathrm{I}$ \\
$E F-2$ & $\mathrm{TN}+\Gamma+\mathrm{I}$ & $\mathrm{LG}+\Gamma$ \\
$r a d A$ & $\mathrm{TIM} 2+\Gamma+\mathrm{I}$ & $\mathrm{LG}+\Gamma$ \\
$r p o B^{\prime}$ & $\mathrm{TN}+\Gamma$ & $\mathrm{LG}+\Gamma$ \\
$\sec Y$ & $\mathrm{TIM} 3+\Gamma$ & $\mathrm{LG}+\Gamma+\mathrm{I}$ \\
\hline
\end{tabular}

All of the models proposed included the gamma shape parameter, and all but $r p o B^{\prime}$ and $\sec Y$ considered invariable sites. The proposed models for the amino acid data of the entire dataset were all LG (Le \& Gascuel, 2008). All of the models recommended the gamma shape parameter, but only half included invariable sites (the concatenated data, AtpB and SecY).

Congruence between phylogenetic signals represented in tree topologies and sequence alignments were evaluated using the approximately unbiased (AU) test (Shimodaira, 2002). Site-wise log-likelihood values of all ML topologies were calculated using RAxML version 7.0.4 and the AU test was calculated using Consel version 0.1 (Shimodaira \& Hasegawa, 2001); the Haloarcula subset was evaluated separately. A $P$-value of 1.00 indicates congruence, whereas a $P$-value of $<0.05$ is considered statistically incongruent. Incongruence between one topology and the sequence data of a different gene indicates a discrepancy between the evolutionary histories of those genes, which could be interpreted as evidence for LGT, though other explanations are possible.

\section{RESULTS AND DISCUSSION}

A major goal of this study was to develop the molecular analysis protocol MLSA for phylogeny and taxonomy of the Halobacteriales to be robust to the known diversity and future discovery of strains, species and genera. To test MLSA effectively as a new molecular protocol for taxonomy of the Halobacteriales, it was necessary to test both narrow and broad taxonomic diversity. Such analysis would determine how precise the method is for differentiating small genetic differences between environmental strains from the same putative species and for identifying phylogenetic clusters as strains organized into species, and species structured into genera. It would also test the primers designed for their ability to amplify a wide diversity of haloarchaea. Therefore, 21 closely related strains from seven recognized species belonging to the genus Haloarcula and 31 additional strains from 23 species with validly published names spanning 13 genera throughout the order Halobacteriales were investigated (Table 1).

\section{Effectiveness of PCR primers}

Members of the order Halobacteriales exhibit extensive sequence evolution among known species and environmental strains. To develop effective PCR primers for analyses, identification of targeted loci common to all taxa necessarily had to strike a balance between using genes conserved enough to develop effective primers and those that are modified enough to be phylogenetically informative. Five loci were targeted for sequence analysis: atp $B, E F-2, \operatorname{rad} A$, $r p o B^{\prime}$ and $\sec Y$. All loci except $a t p B$ had at least one allele for which the sequence could not be obtained, no matter how many attempts were made. PCR and sequencing success was improved by the addition of polylinkers or M-13 sequencing primers to the $5^{\prime}$ end, probably due to increased stability of the primers during amplification annealing steps, and exact primer matches (e.g. no degeneracy) during sequencing.

It is not known why some loci were amplified and sequenced more successfully. Primer design could account for some aspects, because attempts to amplify all loci across a diverse group like the Halobacteriales can be difficult. Attempts were made to limit the primer degeneracy to $16 \times$; however, some primer sets that were more degenerate worked better. LGT could also play a role if a homologue was acquired recently from a distant, non-Halobacteriales relative. However, both of those explanations seem unsatisfactory, because they do not clarify why, within the genus Haloarcula, for example, there was differential amplification success for alleles that one would expect to be highly similar. Nonetheless, a $100 \%$ success rate is not necessary to analyse and classify new strains effectively using the MLSA approach, as demonstrated below.

\section{Phylogenetic reconstruction of Haloarcula species}

Three phylogenetic trees with 100 bootstrap pseudoreplicates were constructed for each gene and the concatenated alignment using different criteria: ML, distance and MP. Results containing nodes with bootstrap values $\geqslant 70 \%$ are mapped onto the ML trees (Fig. 1). The ML trees for the Haloarcula subset were built with the GTR substitution model and the gamma shape parameter, with invariable sites included when recommended by the jModelTest results. The distance and MP trees were constructed using the models proposed by the jModelTest.

It has been suggested that concatenation of multiple loci in phylogenetic reconstruction overcomes many of the limitations of single-gene analyses and therefore defines relationships more robustly among taxa for classification purposes (Hanage et al., 2005). Visual inspection of the Haloarcula concatenated alignment tree reveals that a majority of the strains and species fall into two well-supported clades, indicated in Fig. 1(a) by filled and shaded bars; subsequently, members of the 'black' and 'grey' clusters were identified and marked similarly in the single-gene tree phylogenies (Fig. 1b-f). Members of the 'black' and 'grey' clusters form monophyletic clusters with strong bootstrap support for a majority of the strains in the individual gene phylogenies; however, there were exceptions. Loci from strains that deviated from the concatenated phylogeny, and from each other, are interpreted to have experienced LGT. Within the 


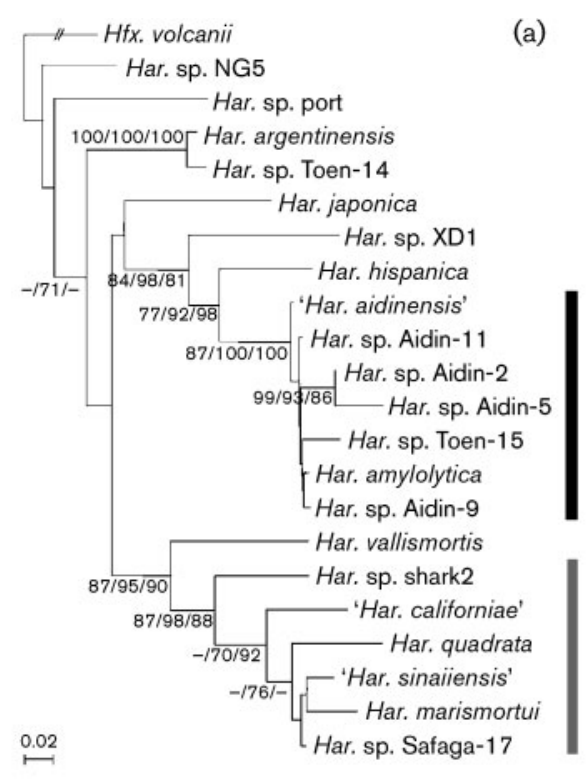

(a)

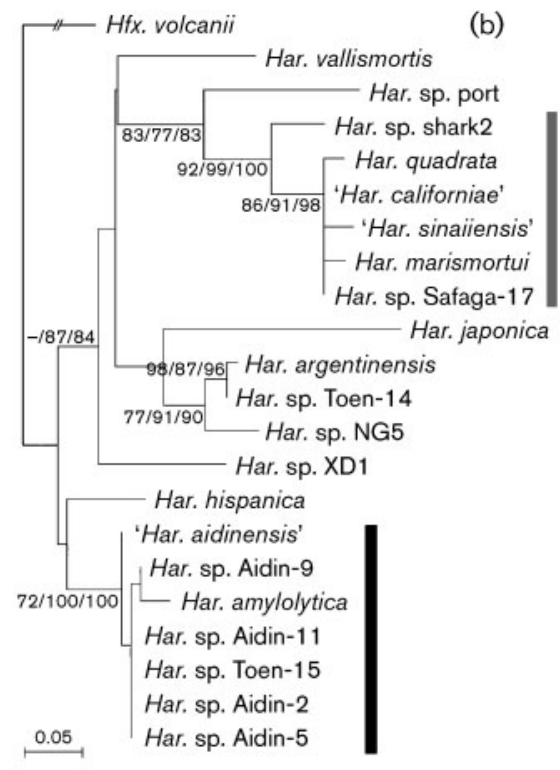

(b)

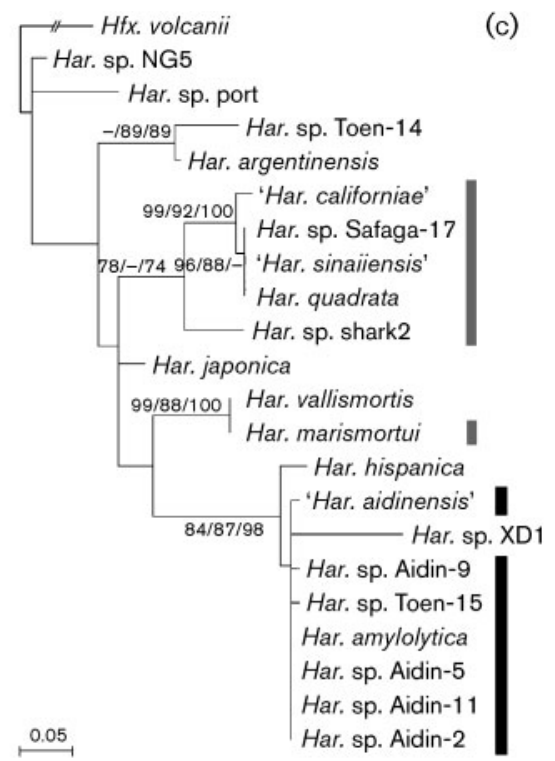

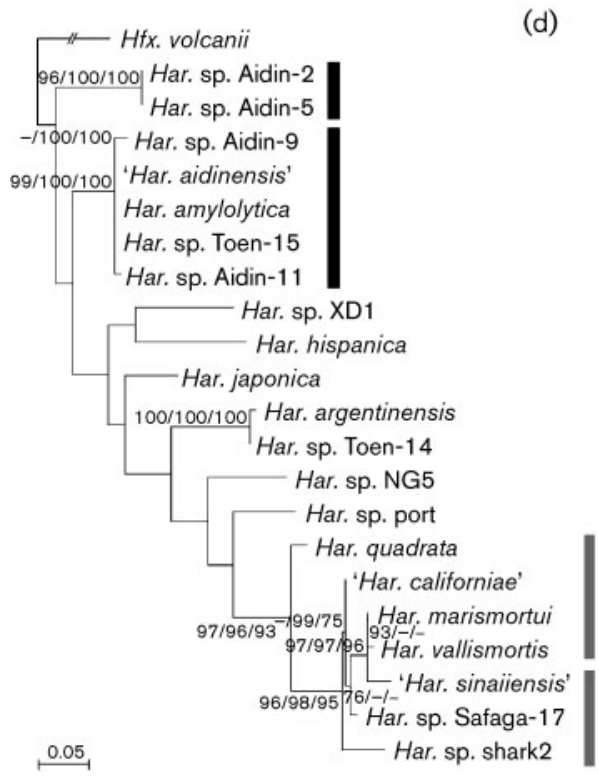

(d)

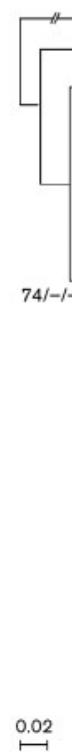

(e)

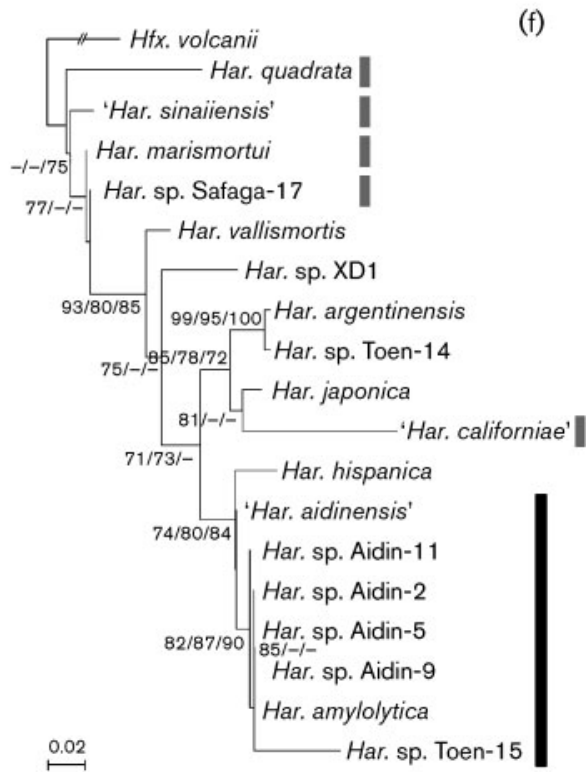

(f)

Fig. 1. Analysis of Haloarcula polarized by Haloferax. ML trees based on nucleotide sequence alignments of a concatenation of all five genes (a), atpB (b), EF-2 (c), radA (d), rpoB' (e) and $\sec Y$ (f). Bootstrap values $\geqslant 70 \%$ were obtained from three separate analyses and are presented in the order ML/distance/MP;,$-<70 \%$. See Table 1 for genus abbreviations, strain details and sequence accession numbers.

'black' and 'grey' clades, there are 'core' strains that remain relatively constant as a cluster, are closely related to each other, are related to Haloarcula amylolytica and Haloarcula marismortui, respectively, often have identical sequences at individual loci and nearly always form a monophyletic group (with exceptions).

Within the 'black' clade, Haloarcula hispanica and Haloarcula sp. XD1 consistently fall outside the 'core', indicating that the MLSA molecular data coincide with the uniqueness of Har. hispanica as being a species separate from Har. amylolytica.
Further, it indicates that Haloarcula sp. XD1 needs to be examined more fully for taxonomic description. Moreover, 'Haloarcula aidinensis', a species whose name has not been validly published, along with each of the 'core' strains of the 'black' clade, clusters together tightly with Har. amylolytica.

The 'grey' clade, however, does not appear as clear-cut in identifying and defining species, probably because of frequent homologous recombination between strains. The major conflicting data are that Har. marismortui and Har. quadrata have nearly identical DNA sequences (see atpB, 
Table 4. AU test results for congruence between loci across Haloarcula strains

Comparisons with a $P$-value $<0.05$ are rejected as incongruent and are in bold.

\begin{tabular}{|lcccccc|}
\hline Dataset & Concatenated & atpB & EF-2 & radA & rpoB' & secY \\
\hline Concatenated & 0.673 & 0.059 & $\mathbf{0 . 0 0 0}$ & $\mathbf{0 . 0 0 0}$ & $\mathbf{0 . 0 0 1}$ & $\mathbf{0 . 0 0 6}$ \\
atpB & $\mathbf{0 . 0 0 0}$ & 0.891 & $\mathbf{0 . 0 0 0}$ & $\mathbf{0 . 0 0 0}$ & $\mathbf{0 . 0 0 1}$ & $\mathbf{0 . 0 0 1}$ \\
EF-2 & $\mathbf{0 . 0 0 0}$ & $\mathbf{0 . 0 0 0}$ & 0.958 & $\mathbf{0 . 0 0 0}$ & $\mathbf{0 . 0 0 0}$ & $\mathbf{0 . 0 0 0}$ \\
radA & $\mathbf{0 . 0 1 6}$ & $\mathbf{0 . 0 0 1}$ & $\mathbf{0 . 0 0 0}$ & 0.927 & $\mathbf{0 . 0 0 0}$ & $\mathbf{0 . 0 0 0}$ \\
rpoB $B^{\prime}$ & 0.149 & $\mathbf{0 . 0 3 1}$ & 0.080 & $\mathbf{0 . 0 3 4}$ & 0.958 \\
secY & 0.557 & 0.277 & 0.086 & 0.135 & $\mathbf{0 . 0 4 9}$ & 0.000 \\
\hline
\end{tabular}

$\left.r p o B^{\prime}\right)$ and so do Har. marismortui and Har. vallismortis (see EF-2 and radA). Furthermore, 'Haloarcula californiae', 'Har. sinaiiensis' and Haloarcula sp. Safaga-17 consistently cluster with either Har. marismortui or Har. quadrata or both; the decision as to which of the two taxonomic species to which they might belong is their correct home is filled with complexity and cannot be sorted out by this analysis. It is clear from this analysis that the different species in the 'grey' clade with validly published names are not genetically isolated; they continue to recombine frequently with other members of the community and form more of a continuum rather than the discrete clusters needed for taxonomic circumscription.

Of the strains that do not fall into the 'black' and 'grey' clusters, only one represents a species with a validly published name (Haloarcula argentinensis). One strain, Toen-14, is closely related to Har. argentinensis and is probably another isolate of that species. However, Haloarcula sp. NG5 and Haloarcula sp. port are sufficiently genetically divergent from all other Haloarcula strains and species that further analysis should be performed to assess their taxonomic standing.

These results demonstrate that MLSA is an effective method for rapid identification and it is sensitive for species circumscription. In this study, the concatenated phylogeny demonstrates clustering patterns that provide important insight into the organization of these strains within the genus Haloarcula, and can address taxonomic questions. Novel species can be identified by comparing the genetic distance of unnamed strains to the type strains of species. For example, Haloarcula sp. port, NG5 and XD1 are sufficiently distant from all known species to warrant their placement within separate species, should additional phenotypic and biochemical data support this claim. Additionally, rapid and inexpensive identification of uncharacterized environmental strains can be carried out within species with validly published names; for example, single-gene and concatenated phylogenies cluster Haloarcula sp. Toen-14 with Har. argentinensis, suggesting a renaming as Har. argentinensis Toen-14.

MLSA also addressed issues of taxonomic uncertainty and raised questions regarding named species. In this analysis, Har. amylolytica and 'Har. aidinensis' were in the same cluster. This cluster encompasses very little sequence divergence, and the number of nucleotide substitutions between Har. amylolytica and 'Har. aidinensis' is only 9 out of 2511 bases analysed. According to Ventosa (2001), 'Har. aidinensis' was proposed as a species, but its name was not validly published because the characterization attempted revealed insufficient differences. Each of the five genes analysed in this study strongly suggests that 'Har. aidinensis' A5 in fact belongs to Har. amylolytica, as do all of the unnamed strains within the 'black' cluster 'core'. Interestingly, this observation suggests that enormous phenotypic or metabolic diversity exists within limited genotypic diversity.

Within the 'grey' cluster, MLSA easily identified two strains as members of separate species, Har. vallismortis and Haloarcula sp. shark2. Har. vallismortis is the type species of the genus; however, the strain shark 2 is remarkably distinct and requires further analysis, as it probably represents a novel taxon. However, there is much more ambiguity within the grey cluster 'core'. Clearly, Har. quadrata and Har. marismortui are different species based on other evidence (Oren et al., 1990, 1999); however, what is not so clear is whether the unnamed 'grey' clade 'core' strains should be divided between them or whether they require their own designations, or possibly that there are no discernible discrete differences because they form a gradient of diversity, with Har. quadrata and Har. marismortui representing the extremes. The major contributing factor to the taxonomic uncertainty is homologous recombination among the strains analysed. This is not a unique situation among the Halobacteriales, as Halorubrum strains also demonstrate this phenomenon (Papke et al., 2004, 2007). A likely scenario is that members of the 'grey' clade core were once a single species in the not too distant past and, through mutation and frequent recombination with each other and with close relatives, they diversified. Rather than demonstrating an easy-to-understand bifurcating evolutionary history, they reveal a network of relationships that complicates their taxonomic placement. However, the observation that cells of 'Har. sinaiiensis' and 'Har. californiae' display a box-shaped morphology suggests they probably belong to the species Har. quadrata, rather than Har. marismortui, which is pleomorphic (Oren et al., 1999).

The single-gene phylogenies show variation between gene histories that can provide insight into species diversification and genus stability. Primarily, the gene phylogenies are incongruent, according to the AU tests (Table 4), probably 
indicating different evolutionary histories. While the 'black' clade and the 'grey' clade are monophyletic in the concatenated phylogeny, they are paraphyletic in many of the single-gene phylogenies. For example, in the EF-2 phylogeny, Haloarcula sp. XD1 is found within the 'black' clade, probably because of an LGT event. Furthermore, in the 'grey' clade, not only is Har. marismortui separate from the rest of the 'grey' clade, but it is also identical to Har. vallismortis, which is probably the result of a more recent LGT event. The $\sec Y$ gene, or portions of it, appears to be highly mobile among the members of the 'grey' clade, as it does not form a cohesive group. Therefore, LGT can be understood as a diversifying force. While there are similar examples of LGT across all of the analysed loci, the majority signal in the concatenated phylogeny strongly supports the cohesion of these clades. This could be due to recombination events occurring more frequently with close relatives, rather than distant ones. The observation that most recombination events within the genus Haloarcula do not come from other genera suggests that LGT, while making some species taxonomy difficult, also acts as a homogenizing force that reinforces genetic cohesiveness at the genus level.

\section{Phylogenetic reconstruction of the Halobacteriales}

Similar to the nucleotide data presented in the analysis of Haloarcula, phylogenetic trees were constructed from the amino acid data under three different criteria: ML, distance and MP. The ML trees were built using the WAG substitution model (Whelan \& Goldman, 2001), the gamma shape parameter and invariable sites when recommended. The distance and MP trees were also constructed using the models predicted by ProtTest. Bootstrap analysis values $\geqslant 70 \%$ from those methodologies were mapped onto the ML trees (Fig. 2). The concatenated phylogeny (Fig. 2a) shows that most species cluster according to their assigned genus. The exceptions to this pattern were strains within the genera Haloterrigena, Natrialba and Natrinema. Species clustered according to genera in all single-gene phylogenies (Fig. 2b-f) except those belonging to the three genera noted above. The only other exception was the genus Halococcus in the EF-2 tree (Fig. 2c), in which Halococcus saccharolyticus appears to have received a gene from a member of Halobacterium. There are also two sets of genera that cluster together (designated by open and filled bars) with significant support in the concatenated phylogeny (Fig. 2a), and these groups coincide with clades I and II, respectively, proposed by Walsh et al. (2004). Members of those clades are similarly identified in the single-gene phylogenies (Fig. 2b-f). Both clades are reconstructed in the RadA and RpoB' phylogenies (Fig. 2d, e); however, only clade I has significant bootstrap support. The species that do not cluster according to genus (see above) are all members of clade I, implying that there is a considerable amount of gene flow among members of the group. The observation that clade I is still observed in the concatenated phylogeny, but is not supported, suggests that its cohesion may be facilitated by biased LGT among its members [e.g. see above and Andam et al. (2010)].

The results of the secY analysis, for both the Haloarcula subset and the Halobacteriales complete set, exhibited significant variation in clustering patterns compared with the other four genes. Interestingly, the $\sec Y$ phylogeny for the Halobacteriales demonstrated that four members of the assumed outgroup (Archaeoglobus fulgidus, Methanosarcina acetivorans, Methanosarcina barkeri and Methanosarcina mazei) clustered within the order Halobacteriales, suggesting a gene transfer from the Halobacteriales. However, there is no bootstrap support for the backbone of the tree, and further investigation is therefore warranted before any firm conclusions should be made. Use of $\sec Y$ in an MLSA set of useful genes appears suboptimal; however, it is too early to make a firm conclusion, and it is nonetheless helpful in understanding gene flow. For instance, use of recombination, like a biological species concept in sexually reproducing organisms, may lead to better species taxonomy. Removal of sec $Y$ from the concatenated datasets had no discernible effect on how strains clustered (not shown).

By investigating a wide range of diversity, the efficacy of MLSA for broad levels of taxonomic organization was explored. Probably the most important aspect of this testing is that the primers designed are capable of amplifying a large number of halobacterial taxa and thus are likely to amplify the entire breadth of known and unknown species. Additionally, generally speaking, each analysed locus, and the concatenated data, organized each of the strains and species into their respective genera and provided an advantage over the 16S rRNA gene for the reasons discussed above (e.g. contain highly divergent paralogous copies, too conserved to differentiate closely related strains and species, intragenically and interspecifically recombinogenic). There was very little statistical support for relationships between genera, except for two groups, which correspond to clades I and II from Walsh et al. (2004). These groups are pronounced in the concatenated phylogeny, but are only replicated in the RadA and RpoB' phylogenies. The cumulative data support organization above the genus level within the Halobacteriales, despite the fact that the individual gene histories provide conflicting results, as indicated by the AU test results (Table 5). Intergeneric recombination appears to be reorganizing the evolutionary past of these genes, but perhaps the residual ancestral signal is still strong enough to capture family-level organization. If additional data continue to support evidence for clades 1 and 2, perhaps a family designation should be applied.

\section{Conclusions}

MLSA as tested proves to be an effective method for reconstructing the phylogeny of the Halobacteriales across taxonomic hierarchical levels. The multilocus approach utilized here was sensitive enough to differentiate closely 

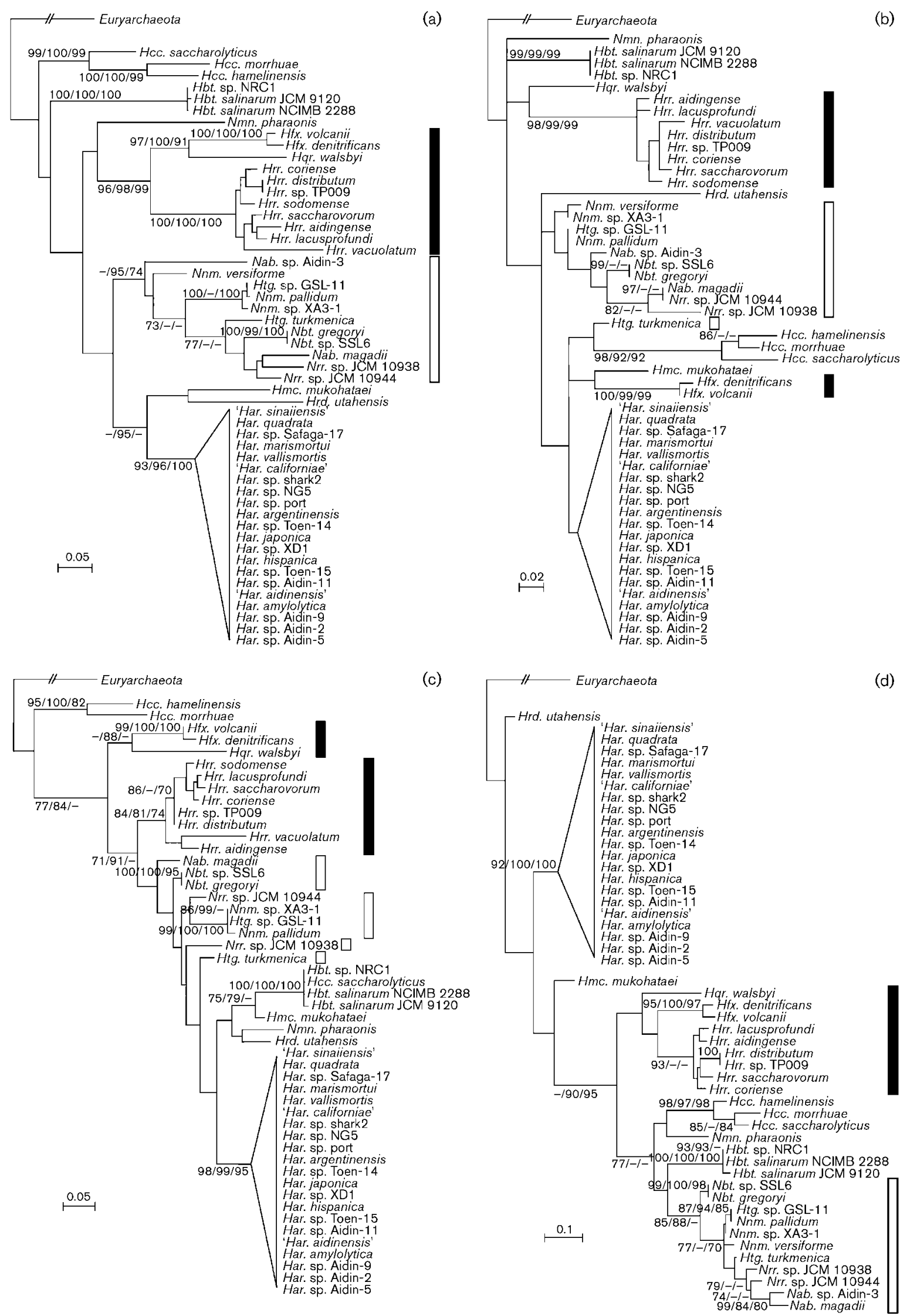

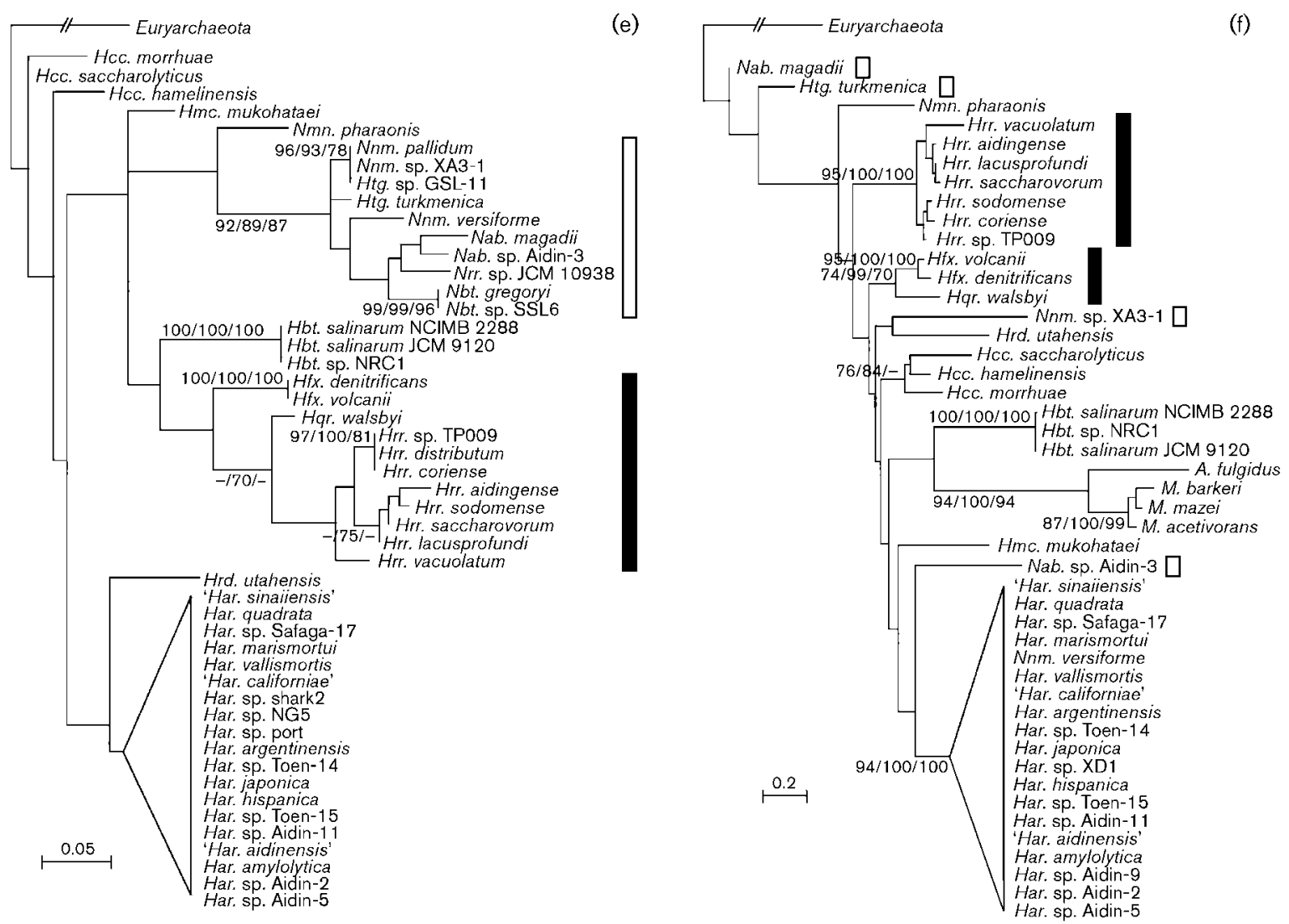

Fig. 2. Analysis of Halobacteriales polarized by euryarchaea. $M L$ trees based on amino acid sequence alignments of a concatenation of all five deduced amino acid sequences (a), AtpB (b), EF-2 (c), RadA (d), RpoB' (e) and SecY (f). Bootstrap values $\geqslant 70 \%$ were obtained from three separate analyses and are presented in the order ML/distance/MP;,$-<70 \%$. See Table 1 for genus abbreviations, strain details and sequence accession numbers. Sequences from six strains were chosen as the outgroup to the Halobacteriales and are referred to as Euryarchaeota in the tree: Methanosarcina acetivorans, Methanosarcina barkeri, Methanosarcina mazei, Archaeoglobus fulgidus, Methanothermobacter thermautotrophicus and Methanococcus vannielii. Genera that formed clades I and II of Enache et al. (2007) and Walsh et al. (2004) are indicated by bars; open bars indicate representatives of clade I and filled bars indicate representatives of clade II. Additional genus abbreviations in (f): A., Archaeoglobus; M., Methanosarcina.

related strains from the same species and to identify strains of sufficient uniqueness that they probably belong to novel species. Furthermore, it could recognize family-like hierarchy within the order. These two attributes, fine-scale sensitivity and broad-scale application, make MLSA a highly versatile method for taxonomy. Furthermore, by analysing multiple strains from the same species, a better understanding of population dynamics, evolutionary processes and the

Table 5. AU test results for congruence between loci across Halobacteriales strains

Comparisons with a $P$-value $<0.05$ are rejected as incongruent and are in bold.

\begin{tabular}{|lcccccc|}
\hline Dataset & Concatenated & AtpB & EF-2 & RadA & RpoB $^{\prime}$ & SecY \\
\hline Concatenated & 0.978 & $\mathbf{0 . 0 0 1}$ & $\mathbf{0 . 0 0 0}$ & $\mathbf{0 . 0 0 0}$ & $\mathbf{0 . 0 0 1}$ & $\mathbf{0 . 0 0 0}$ \\
AtpB & $\mathbf{0 . 0 0 0}$ & 0.901 & $\mathbf{0 . 0 0 0}$ & $\mathbf{0 . 0 0 0}$ & $\mathbf{0 . 0 0 0}$ \\
EF-2 & $\mathbf{0 . 0 0 0}$ & 0.082 & 0.984 & $\mathbf{0 . 0 0 4}$ & 0.069 \\
RadA & $\mathbf{0 . 0 0 6}$ & 0.160 & $\mathbf{0 . 0 3}$ & 0.929 & 0.258 \\
RpoB' & $\mathbf{0 . 0 0 2}$ & 0.193 & $\mathbf{0 . 0 3 1}$ & $\mathbf{0 . 0 2 9}$ & 0.901 \\
SecY & $\mathbf{0 . 0 4 1}$ & 0.240 & $\mathbf{0 . 0 1 1}$ & 0.145 & 0.073 \\
\end{tabular}


biological relevance of operational taxonomic units and species becomes accessible. For instance, examining multiple strains that comprised the 'black' and 'grey' clades in the genus Haloarcula led to fruitful insight into their taxonomic standing. Additionally, through the examination of tens of Halorubrum strains, Papke et al. (2007) demonstrated the existence of phylogenetic clusters in which recombination was more prevalent within than between clusters, suggesting a quasi-biological species concept. Thus, application of MLSA to the detection of recombination in the Halobacteriales could facilitate an important, currently unrecognized character for species circumscription. However, irrespective of recombination, MLSA in general provides more insight into the dynamics of speciation and species evolution, which are critical for stable species nomenclature.

There has been growing recognition of the power of MLSA for epidemiological, ecological and taxonomic practices. This method is rapid and portable and generates storable data that are highly sensitive and phylogenetically relevant (Maiden et al., 1998; Urwin \& Maiden, 2003). It has been suggested that MLSA could be an effective tool to supplement or even supplant DNA-DNA hybridization (DDH), which does not generate storable data, for species circumscription in the recommended polyphasic approach to taxonomy (Young et al., 2008). Of course, more comparative analyses between MLSA and DDH need to be done before such a recommendation should be made for its use in the Halobacteriales.

\section{ACKNOWLEDGEMENTS}

We wish to acknowledge the following agencies for funding this research: National Science Foundation (award nos 0919290 and 0830024), the US-Israel Binational Science Foundation (award no. 2007043), the Spanish Ministry of Education and Science (GL201019303) and the Junta de Andalucia (10-CVI-6226). We also wish to acknowledge the following people for generously supplying their isolates: Drs Haruo Saida, Takashi Nagai and Humitake Seki of the University of Tsukuba, Japan; Dr Zhou Peijin of the Institute of Microbiology, China; Dr Hiroaki Tomioka of Saitama University, Japan; and Dr Vivek Upasani of the M. G. Science Institute, India.

\section{REFERENCES}

Abascal, F., Zardoya, R. \& Posada, D. (2005). ProtTest: selection of best-fit models of protein evolution. Bioinformatics 21, 2104-2105.

Andam, C. P., Williams, D. \& Gogarten, J. P. (2010). Natural taxonomy in light of horizontal gene transfer. Biol Philos 25, 589-602.

Asker, D. \& Ohta, Y. (2002). Haloferax alexandrinus sp. nov., an extremely halophilic canthaxanthin-producing archaeon from a solar saltern in Alexandria (Egypt). Int J Syst Evol Microbiol 52, 729-738.

Bodaker, I., Sharon, I., Suzuki, M. T., Feingersch, R., Shmoish, M., Andreishcheva, E., Sogin, M. L., Rosenberg, M., Maguire, M. E. \& other authors (2010). Comparative community genomics in the Dead Sea: an increasingly extreme environment. ISME J 4, 399-407.

Boucher, Y., Douady, C. J., Sharma, A. K., Kamekura, M. \& Doolittle, W. F. (2004). Intragenomic heterogeneity and intergenomic recombination among haloarchaeal rRNA genes. J Bacteriol 186, 3980-3990.
Casamayor, E. O., Massana, R., Benlloch, S., Øvreås, L., Diez, B., Goddard, V. J., Gasol, J. M., Joint, I., Rodríguez-Valera, F. \& PedrósAlió, C. (2002). Changes in archaeal, bacterial and eukaryal assemblages along a salinity gradient by comparison of genetic fingerprinting methods in a multipond solar saltern. Environ Microbiol 4, 338-348.

Case, R. J., Boucher, Y., Dahllöf, I., Holmström, C., Doolittle, W. F. \& Kjelleberg, S. (2007). Use of $16 \mathrm{~S}$ rRNA and $r p o B$ genes as molecular markers for microbial ecology studies. Appl Environ Microbiol 73, 278-288.

Cuadros-Orellana, S., Martin-Cuadrado, A. B., Legault, B., D'Auria, G., Zhaxybayeva, O., Papke, R. T. \& Rodriguez-Valera, F. (2007). Genomic plasticity in prokaryotes: the case of the square haloarchaeon. ISME J 1, 235-245.

Edgar, R. C. (2004a). MUSCLE: a multiple sequence alignment method with reduced time and space complexity. BMC Bioinformatics 5, 113.

Edgar, R. C. (2004b). MUSCLE: multiple sequence alignment with high accuracy and high throughput. Nucleic Acids Res 32, 1792-1797.

Enache, M., Itoh, T., Fukushima, T., Usami, R., Dumitru, L. \& Kamekura, M. (2007). Phylogenetic relationships within the family Halobacteriaceae inferred from $r p o B^{\prime}$ gene and protein sequences. Int J Syst Evol Microbiol 57, 2289-2295.

Guindon, S. \& Gascuel, O. (2003). A simple, fast, and accurate algorithm to estimate large phylogenies by maximum likelihood. Syst Biol 52, 696-704.

Hanage, W. P., Fraser, C. \& Spratt, B. G. (2005). Fuzzy species among recombinogenic bacteria. BMC Biol 3, 6 .

Kamaishi, T., Hashimoto, T., Nakamura, Y., Nakamura, F., Murata, S., Okada, N., Okamoto, K., Shimizu, M. \& Hasegawa, M. (1996). Protein phylogeny of translation elongation factor EF-1 alpha suggests microsporidians are extremely ancient eukaryotes. $J \mathrm{Mol}$ Evol 42, 257-263.

Le, S. Q. \& Gascuel, O. (2008). An improved general amino acid replacement matrix. Mol Biol Evol 25, 1307-1320.

Legault, B. A., Lopez-Lopez, A., Alba-Casado, J. C., Doolittle, W. F., Bolhuis, H., Rodriguez-Valera, F. \& Papke, R. T. (2006). Environmental genomics of "Haloquadratum walsbyi" in a saltern crystallizer indicates a large pool of accessory genes in an otherwise coherent species. BMC Genomics 7, 171.

López-López, A., Benlloch, S., Bonfá, M., Rodríguez-Valera, F. \& Mira, A. (2007). Intragenomic $16 \mathrm{~S}$ rDNA divergence in Haloarcula marismortui is an adaptation to different temperatures. J Mol Evol 65, 687-696.

Maddison, W. P. \& Maddison, D. R. (2009). Mesquite: a modular system for evolutionary analysis, version 2.71 . http://mesquiteproject. org.

Maiden, M. C., Bygraves, J. A., Feil, E., Morelli, G., Russell, J. E., Urwin, R., Zhang, Q., Zhou, J., Zurth, K. \& other authors (1998). Multilocus sequence typing: a portable approach to the identification of clones within populations of pathogenic microorganisms. Proc Natl Acad Sci U S A 95, 3140-3145.

Minegishi, H., Kamekura, M., Itoh, T., Echigo, A., Usami, R. \& Hashimoto, T. (2010). Further refinement of the phylogeny of the Halobacteriaceae based on the full-length RNA polymerase subunit B' (rpoB') gene. Int J Syst Evol Microbiol 60, 2398-2408.

Nakamura, L. K., Roberts, M. S. \& Cohan, F. M. (1999). Relationship of Bacillus subtilis clades associated with strains 168 and W23: a proposal for Bacillus subtilis subsp. subtilis subsp. nov. and Bacillus subtilis subsp. spizizenii subsp. nov. Int J Syst Bacteriol 49, 1211-1215.

Ochsenreiter, T., Pfeifer, F. \& Schleper, C. (2002). Diversity of Archaea in hypersaline environments characterized by molecularphylogenetic and cultivation studies. Extremophiles 6, 267-274. 
Oren, A. (1994). The ecology of the extremely halophilic archaea. FEMS Microbiol Rev 13, 415-439.

Oren, A. (2008). Microbial life at high salt concentrations: phylogenetic and metabolic diversity. Saline Syst 4, 2.

Oren, A. \& Ventosa, A. (2000). International Committee on Systematic Bacteriology Subcommittee on the taxonomy of Halobacteriaceae. Minutes of the meetings, 16 August 1999, Sydney, Australia. Int J Syst Evol Microbiol 50, 1405-1407.

Oren, A., Ginzburg, M., Ginzburg, B. Z., Hochstein, L. I. \& Volcani, B. E. (1990). Haloarcula marismortui (Volcani) sp. nov., nom. rev., an extremely halophilic bacterium from the Dead Sea. Int J Syst Bacteriol 40, 209-210.

Oren, A., Ventosa, A., Gutiérrez, M. C. \& Kamekura, M. (1999). Haloarcula quadrata sp. nov., a square, motile archaeon isolated from a brine pool in Sinai (Egypt). Int J Syst Bacteriol 49, 1149-1155.

Papke, R. T. (2009). A critique of prokaryotic species concepts. Methods Mol Biol 532, 379-395.

Papke, R. T., Douady, C. J., Doolittle, W. F. \& Rodríguez-Valera, F. (2003). Diversity of bacteriorhodopsins in different hypersaline waters from a single Spanish saltern. Environ Microbiol 5, 1039-1045.

Papke, R. T., Koenig, J. E., Rodríguez-Valera, F. \& Doolittle, W. F. (2004). Frequent recombination in a saltern population of Halorubrum. Science 306, 1928-1929.

Papke, R. T., Zhaxybayeva, O., Feil, E. J., Sommerfeld, K., Muise, D. \& Doolittle, W. F. (2007). Searching for species in haloarchaea. Proc Natl Acad Sci U S A 104, 14092-14097.

Pasić, L., Ulrih, N. P., Crnigoj, M., Grabnar, M. \& Velikonja, B. H. (2007). Haloarchaeal communities in the crystallizers of two adriatic solar salterns. Can J Microbiol 53, 8-18.

Posada, D. (2008). jModelTest: phylogenetic model averaging. Mol Biol Evol 25, 1253-1256.

Renesto, P., Gouvernet, J., Drancourt, M., Roux, V. \& Raoult, D. (2001). Use of $r p o B$ gene analysis for detection and identification of Bartonella species. J Clin Microbiol 39, 430-437.

Sandler, S. J., Hugenholtz, P., Schleper, C., DeLong, E. F., Pace, N. R. \& Clark, A. J. (1999). Diversity of radA genes from cultured and uncultured archaea: comparative analysis of putative RadA proteins and their use as a phylogenetic marker. J Bacteriol 181, 907-915.

Sheppard, S. K., McCarthy, N. D., Falush, D. \& Maiden, M. C. (2008). Convergence of Campylobacter species: implications for bacterial evolution. Science 320, 237-239.

Shimodaira, H. (2002). An approximately unbiased test of phylogenetic tree selection. Syst Biol 51, 492-508.

Shimodaira, H. \& Hasegawa, M. (2001). CONSEL: for assessing the confidence of phylogenetic tree selection. Bioinformatics 17, 1246-1247.

Stamatakis, A. (2006). RAxML-VI-HPC: maximum likelihood-based phylogenetic analyses with thousands of taxa and mixed models. Bioinformatics 22, 2688-2690.

Swofford, D. L. (2003). PAUP*: phylogenetic analysis using parsimony, version 4.0b10. Sunderland, MA: Sinauer Associates.

Tamura, K. \& Nei, M. (1993). Estimation of the number of nucleotide substitutions in the control region of mitochondrial DNA in humans and chimpanzees. Mol Biol Evol 10, 512-526.

Tavaré, S. (1986). Some probabilistic and statistical problems in the analysis of DNA sequences. In Lectures on Mathematics in the Life Sciences (Some Mathematical Questions in Biology), vol. 17, pp. 57-86. Edited by R. M. Miura. Providence, RI: American Mathematical Society.

Urwin, R. \& Maiden, M. C. (2003). Multi-locus sequence typing: a tool for global epidemiology. Trends Microbiol 11, 479-487.

Ventosa, A. (2001). Genus II. Haloarcula. In Bergey's Manual of Systematic Bacteriology, 2nd edn, vol. 1, pp. 305-309. Edited by D. R. Boone, R. W. Castenholz \& G. M. Garrity. New York: Springer.

Walsh, D. A., Bapteste, E., Kamekura, M. \& Doolittle, W. F. (2004). Evolution of the RNA polymerase $\mathrm{B}^{\prime}$ subunit gene $\left(r p o B^{\prime}\right)$ in Halobacteriales: a complementary molecular marker to the SSU rRNA gene. Mol Biol Evol 21, 2340-2351.

Whelan, S. \& Goldman, N. (2001). A general empirical model of protein evolution derived from multiple protein families using a maximum-likelihood approach. Mol Biol Evol 18, 691-699.

Young, J. M., Park, D. C., Shearman, H. M. \& Fargier, E. (2008). A multilocus sequence analysis of the genus Xanthomonas. Syst Appl Microbiol 31, 366-377. 